\title{
Editorial: Optoelectronic Properties of Two-Dimensional Systems
}

\author{
S. Forti ${ }^{1 *}$, A. Principi $^{2 *}$ and D. Bandurin ${ }^{3 *}$ \\ ${ }^{1}$ Center for Nanotechnology Innovation IIT@NEST, Pisa, Italy, ${ }^{2}$ Department of Physics and Astronomy, The University of \\ Manchester, Manchester, United Kingdom, ${ }^{3}$ Department of Physics, Massachusetts Institute of Technology, Cambridge, MA, \\ United States
}

Keywords: optoelecronics, graphene, electron micoscopy, band structure, ultrafast dynamics, 2D materials, photoelectron spectroscopy

\section{Editorial on the Research Topic}

Optoelectronic Properties of Two-Dimensional Systems

Two-dimensional (2D) materials are emerging as the new frontier of research in solid state physics. The downscaling of electronic devices, in both size and energy consumption, passes through the improvement of fabrication techniques and the combination of 2D materials in van der Waals ( $\mathrm{vdW}$ ) heterostructures [1]. In recent years, more and more materials have been synthesized, whose very diverse properties have been analyzed with various techniques [2-4]. Stacking 2D materials together, and controlling the relative twist angle with an accuracy of less than a tenth of a degree [5], has opened an avenue for producing artificial materials with properties defined "on demand". The improvement and refinement of transfer techniques play a key role in determining the quality of the resulting vdW structure, and so do the ample progress made by the development of large-scale production techniques, such as chemical vapor deposition (CVD) [6].

The research topic Optoelectronic Properties of Two-Dimensional Systems presents some relevant

\section{OPEN ACCESS}

Edited and reviewed by: James Avery Sauls, Northwestern University, United States

${ }^{*}$ Correspondence: S. Forti stiven.forti@it.it

A. Principi

alessandro.principi@ manchester.ac.uk

D. Bandurin

bandurin@mit.edu

Received: 10 November 2021 Accepted: 30 November 2021 Published: 21 December 2021

Citation:

Forti S, Principi $A$ and Bandurin D (2021) Editorial: Optoelectronic Properties of TwoDimensional Systems. Front. Phys. 9:812601. doi: 10.3389/fphy.2021.812601 contribution to the investigation of the optoelectronic properties of $2 \mathrm{D}$ materials and vdW stacks.

In the work of Zakharov, the properties of epitaxial graphene on $6 \mathrm{H}-\mathrm{SiC}(0001)$ are described via low-energy electron microscopy (LEEM) measurements, under the condition of having one or two layers of $\mathrm{Ge}$ atoms intercalated at the heterointerface between graphene and SiC. Zakharov finds that Ge atoms on the second Ge layer diffuse much faster and so the mono-vs. bi-layer distribution can be controlled by temperature. In turn, this means that atomically sharp graphene $p / n$ junctions can be realized in a controlled manner [7], opening the way for potential applications in pseudo-spin electronics and electron optics.

The article led by Prof. Flege et al. makes again use of LEEM and X-ray photoemission electron microscopy (XPEEM) to monitor the transition from one to two layers in $\mathrm{MoS}_{2}$ grown on $\mathrm{Au}$ (111), showing the microscopic mechanism through which the second layer forms and providing valuable indications for synthetizing pure single layer molybdenum disulfide, a $1.83 \mathrm{eV}$ direct bandgap semiconductor with remarkable optical properties.

In the article of Krause et al., the authors look at the quasiparticle dynamics in the bilayer $\mathrm{WS}_{2} /$ graphene vdW heterostructure. Time- and angle-resolved photoemission spectroscopy (Tr-ARPES) is a refined and powerful tool providing insightful information about the dynamics of transient states of the carriers excited from the valence to the conduction band. The authors use this technique to investigate the ultrafast charge transfer between graphene and $\mathrm{WS}_{2}$, showing that the indirect nature of the bandgap in the $\mathrm{WS}_{2}$ bilayer does not hinder the charge transfer mechanisms between the two $2 \mathrm{D}$ materials.

A relevant theoretical contribution to the description of the electron-phonon scattering is provided by the work of Narozhny and Gornyi, in which they model the supercollision mechanism in a regime of weak-violation of the energy conservation, showing that at very high temperature, supercollision represents the dominant decay channel. 
The Optoelectronic Properties of Two-Dimensional Systems is therefore a Research Topic that touches multiple aspects of the $2 \mathrm{D}$ materials, from synthesis to structure, from optical to electronic properties and to theoretical modelling.

\section{REFERENCES}

1. Geim AK, Grigorieva IV Van Der Waals Heterostructures. Nature (2013) 499: 419-25. doi:10.1038/nature12385

2. Sierra JF, Fabian J, Kawakami RK, Roche S, Valenzuela SO Van Der Waals Heterostructures for Spintronics and Opto-Spintronics. Nat Nanotechnol (2021) 16:856-68. doi:10.1038/s41565-021-00936-x

3. Dong J, Zhang L, Dai X, Ding F The Epitaxy of 2D Materials Growth. Nat Commun (2020) 11:5862. doi:10.1038/s41467-020-19752-3

4. Liu Y, Weiss NO, Duan X, Cheng HC, Huang Y, Duan X Van Der Waals Heterostructures and Devices. Nat Rev Mater (2016) 1:16042. doi:10.1038/ natrevmats.2016.42

5. Cao Y, Fatemi V, Fang S, Watanabe K, Taniguchi T, Kaxiras E, et al. Unconventional Superconductivity in Magic-Angle Graphene Superlattices. Nature (2018) 556:43-50. doi:10.1038/nature26160

6. Pace S, Martini L, Convertino D, Keum D, Forti S, Pezzini S, et al. Synthesis Of Large-Scale Monolayer $1 T^{\prime}-\mathrm{MoTe}_{2}$ and its Stabilization Via Scalable hBN Encapsulation. ACS Nano (2021) 15:4213. doi:10.1021/acsnano.0c05936

\section{AUTHOR CONTRIBUTIONS}

All authors listed have made a substantial, direct, and intellectual contribution to the work and approved it for publication.

7. Baringhaus J, Stöhr A, Forti S, Krasnikov SA, Zakharov AA, Starke U, et al. Bipolar Gating of Epitaxial Graphene by Intercalation of Ge. Appl Phys Lett (2014) 104:261602. doi:10.1063/1.4886411

Conflict of Interest: The authors declare that the research was conducted in the absence of any commercial or financial relationships that could be construed as a potential conflict of interest.

Publisher's Note: All claims expressed in this article are solely those of the authors and do not necessarily represent those of their affiliated organizations, or those of the publisher, the editors and the reviewers. Any product that may be evaluated in this article, or claim that may be made by its manufacturer, is not guaranteed or endorsed by the publisher.

Copyright (C) 2021 Forti, Principi and Bandurin. This is an open-access article distributed under the terms of the Creative Commons Attribution License (CC BY). The use, distribution or reproduction in other forums is permitted, provided the original author(s) and the copyright owner(s) are credited and that the original publication in this journal is cited, in accordance with accepted academic practice. No use, distribution or reproduction is permitted which does not comply with these terms. 